Finally, I think it should be put privately to the Prime Minister that the profession and many patients have completely lost confidence in Mrs Castle and that her resignation would be of great benefit to the community as a whole.

Birmingham

J A G PARIS

SIR,-At a meeting on Monday 1 December 1975 of 27 members of the consultant staff of South-east Kent District it was agreed that the BMA recommendation that consultants should undertake only emergency work and maintain a service to cancer patients, sick children, obstetrics, and inpatients be fully implemented. The issue is not only of private beds in hospitals but of the freedom of doctor and patient to select an alternative to the NHS. The most recent statement by the Secretary of State refers to limitation of private practice outside the Health Service. No other employee has such restriction placed on the use of his spare time.

The wish of the consultant staff is that the entire functioning of the NHS and not just part of it should be referred to the royal commission and in the interests of freedom of the entire population they must maintain their withdrawal until this simple request is agreed.

All consultants deeply regret the inconvenience to the public but feel it is due to the intransigence of the Secretary of State in failing to agree to independent judgment.

\section{H NELSON} E B LEWIS Secretary,

Folkestone, Kent Joint Medical Staff Committee,
South-east Kent Health District

SIR,-We now find ourselves involved in industrial action which, while we considered it essential, is odious to all. Nevertheless, there has not emerged among junior doctors a generally accepted consensus of opinion as to our aim. Surely we are not causing such inconvenience and, increasingly, hardship to the sick solely to get more money for ourselves. Yet this is the impression which a few doctors have tended to give and which is conveyed to the public in the lay press.

Rather we must confirm to ourselves, and affirm to the Government and public, that our aim is to improve the quality of care we give our patients. To this end we should not seek more money to pay those doctors who work in excess of 80 hours a week but state categorically that the maximum hours anyone will work is 80 . We cannot work more hours if we are to provide the quality of medicine our patients need and deserve.

If we were to take this stand the Department of Health and Social Security would be compelled to distribute its resources in such a manner that the quality of care necessary could be provided for those who are most in need. It would also facilitate the introduction of our new-style contract without the need for a no-detriment clause.

Coventry

HUGH ANNETT

SIR,-We are both members of the BMA and wish to remain so, although we cannot St Leonards-on-Sea, Sussex enact its recommendations for the junior hospital doctors in the present dispute.

It would seem to us that suoh action would increase the morbidity and mortality of patients under our care and that where such action is being taken patients are undoubtedly suffering. We feel that the medical profession has resigned all sanity on the present issue and implore our negotiating representatives to extricate us from this chaos and to state our reasonable claims in moderate and professional voice without forcing us to ransom our patients.

Queen Elizabeth II Hospital,

FRANCES ROCHFORD Welwyn Garden City, Herts

Lewisham Hospital,

R F MASSEY

SIR,-Well, here we are in the thick of "industrial action," and does it not stink? That we are doing wrong is obvious: patients are suffering and many will die as a result of our action. Not only are we breaking an ethical code that should be our very backbone but we are also going directly against the elected Government. And what is worse is that our democratic ballot caused it. It is a lesson for us all to learn-that democracy is not always right, the majority can get the answer wrong. It is a pity the BMA had to take heed of the ballot and did not advise us against this folly.

It is to be hoped that, at last, the rest of the country may see how disastrous strike action is, how much suffering it causes, how it holds innocent people to ransom. It is interesting to see how we can be misled into this course of action by our apathy and discontent. It is interesting to see, as happens in so many other disputes, how one group of our workforce can force others into toeing the line-our anaesthetists, for example.

Resignation is the only ethical alternative, apart from a steady united noise; if we feel strongly enough about our pay or our private patients, then we should resign. But if we do not feel strongly enough about it to resign we should certainly not be harming our patients instead.

S R BRENNAN

Royal Hospital

SIR,-Consultants have had their contractual increments frozen, itself a great injustice, and some have received only part of the pay increase promised last April. Could not these funds, owed in equity to our profession, be used to make no-detriment payments to the junior staff without breaching the pay code?

It is well known that some juniors stand to lose several hundred pounds a year under the new contracts for doing the same amount of work. It is hypocritical of the Government to preach about the sanctity of the pay code while putting individuals into this position. It seems to me that "no detriment" is a much better issue for the juniors to fight on than additional overtime payments, which are already getting them a bad press.

No one should be expected to work more than 80 hours a week and hospital work should be organised to make this unnecessary.

R E IRVINE

\section{Metal sensitivity in patients with joint prostheses}

SIR,-I do congratulate the authors of the two articles on this subject (15 November, pp 374 and 376) on the pains and trouble they have taken and the extensive investigations they have carried out. I am sure it all has some relevance in total replacement operations where metal is used as a bearing surface, but one must obtain a balanced outlook. There is an enormous field where chrome cobalt alloy implants have been used over many years and yet the cases of metal sensitivity have been conspicuous by their rarity. There has been one case reported from Norwich ${ }^{1}$ of sensitivity where chrome cobalt was used simply as an implant, and this is over a period of some 35 years in which it has been used in literally tens of thousands of cases. It is to be expected that a somewhat higher incidence might occur in a working joint replacement because a certain amount of wear must occur and finely divided particles would collect around the joint. However, in a series of over 1000 cases at Norwich and over a period of 20 years there has only been one case of metal sensitivity with clinical manifestations recorded, and in this case the hip replacement has remained firm and satisfactory. The patient is still a practising doctor and plays golf, and it is over six years now since the operation took place so it can be safely claimed that no loosening of the components has occurred.

There have been several articles recently suggesting that loosening and giving way of the medial wall of the acetabulum are due to cobalt toxicity, but this conclusion is certainly not borne out by an analysis of my cases. In a consecutive series of 700 cases with a minimum follow-up of four years and a maximum of 14 years there were nine cases in which the medial wall of the acetabulum had given way. They were all in female patients. Four of them recovered with restricted activities and are now in the "excellent" category, and there were five cases that required surgery, but only two of these required a Girdlestone's operation; and they all had obvious defects such as trauma or faults in operative technique to account for the loosening and giving way of the acetabulum. If they had been due to cobalt toxicity one would have expected it to continue to act detrimentally after the conservative treatment or revision procedure. Five of these patients were patch-tested. Two were strongly positive to cobalt, three were weakly positive to cobalt, and two were strongly positive to nickel, but all these were among the ones who recovered well.

It is easy to draw hasty and inaccurate conclusions from this question of metal sensitivity, and I feel one can reassure patients who have had a metal-on-metal prosthesis inserted that they are most unlikely to have any trouble from metal toxicity. The fact that all my patients with loosening and giving way of the acetabulum were female would suggest that osteoporotic bone is more likely to be a major underlying factor. The only patient in my experience of the use of these metal-on-metal joints who had any clinical evidence of metal sensitivity was a man, and he has had no trouble from his total hip replacement, which has been in for over six years. I am preparing a special article to deal with this subject in 
more detail, but I feel that a reassurance to patients who have had this most successful procedure carried out is necessary at this stage. Norfolk and Norwich Hospital,
Norwich, Norfolk

1 Mckenzie, A W, Aitken, C V E, and Ridsdill-

SIR,-We read with interest the papers of Mr M K D Benson and others and Dr M W Elves and others (15 November, pp 374 and 376 respectively). We can confirm that since our paper on the subject of cobalt toxicity in relation to $\mathrm{McKee}$ hip arthroplasty ${ }^{1}$ was published we also have found that approximately one-third of patients with a metal to metal (cobalt-chrome-molybdenum alloy) prostheses in situ will give a positive reaction to one of the metals of skin testing.

Of 20 patients so far reviewed, four are cobalt-positive, two nickel-positive, and one cobalt- and nickel-positive. The significance of a positive patch test remains unproved, however, and neither of the articles you have published have provided sufficient histological, immunological, or radiological evidence to permit any firm conclusion. Of the patients reviewed by us, there have been several with a positive skin patch test and yet perfect hip function. This is also the experience in at least one other centre.

There are at least two points arising which merit further discussion. Firstly, metallic cobalt and cobalt salts are not only irritants but are directly toxic and this in itself may be significant in the production of any necrotic reaction around the joint. Also, polymethacrylate cement is not beyond suspicion as a possible cause of necrotic reaction. It is important that conclusions such as that of $\mathrm{Mr}$ Benson and his colleagues that "it therefore seems advisable to use Charnley or other forms of metal-to-plastic prostheses in preference to metal-to-metal ones" should not be made on the basis of a statistical relationship between skin sensitivity and loosening of the prostheses, particularly when only two cases of loosening are mentioned, neither of which is described nor is the orthopaedic evidence for loosening presented. We are more in agreement with Dr Elves and his colleagues and the guarded conclusion in their paper.

Secondly, we feel it is of great importance that authors on this subject state clearly which metal is used in the metal-to-plastic joints. Thus the femoral component of the Charnley prostheses is produced in stainless steel (for example, Thackray) and cobalt chrome alloy (for example, Down Bros). It may be that it is the use of stainless steel articulating with high-density polyethylene that leads to a low rate of increased metal sensitivity rather than the fact that it is a metal-to-plastic joint. We would therefore ask all orthopaedic surgeons who use the metal-to-plastic prostheses to document clearly in their case notes the particular material used in the femoral component as this may be of great significance at a later date. In this centre the information is being documented in the theatre register.

\section{ANTHONY JONES} KEITH LUCAS

Bristol Royal Infirmary and Winford Orthopaedic Hospital, Bristol
Jones, D A, et al, fournal of Bone and foint
Surgery, 1975, 57B, 294. Ring, P A, personal communication, 1974

\section{Use of clonazepam in epilepsy}

SIR,-In his article on epilepsy ( 1 November, p 270) Dr F B Gibberd did not mention the use of the newer benzodiazepine anticonvulsant clonazepam, which has been shown to be effective both in the control of seizures in childhood ${ }^{1}$ and in intractable epilepsy. ${ }^{2}$ This drug may also be useful in controlling status epilepticus without resort to the use of anaesthesia, muscle relaxants, and positive pressure ventilation, as the following brief clinical summary illustrates.

A 6-year-old boy with epilepsy and mental handicap following encephalitis at 4 months of age was admitted in status epilepticus in February 1975. Seizures were well controlled with intravenous diazepam and intramuscular paraldehyde for four days. Thereafter they recurred despite the continuation of this therapy, and treatment with intravenous clonazepam was started. An initial dose of $3 \mathrm{mg}$ in a $500-\mathrm{ml}$ infusion of $5 \%$ dextrose was ineffective, but by gradually increasing the dose over the next 48 hours the seizures were controlled. A total of $43 \mathrm{mg}$ of clonazepam was infused. Despite this high dose there was no evidence of respiratory depression, hypotonia, or excessive bronchial secretion. Subsequently the dose was reduced and good control was maintained with $3 \mathrm{mg}$ daily by mouth in combination with phenobarbitone.

Although further experience with clonazepam is required, it may have a useful place in the control of status epilepticus, as previous reports have suggested. ${ }^{34}$

HENRY L HALLIDAY JOHN F T GLASGOW

Royal
Belfas

1 Martin, D, and Hirt, H R, Neuropädiatrie, 1973, 4, 245 .
Hooshmand, H, Archives of Neurology, 1972, 27, 205.

3 Gastaut, H, et al, Epilepsia, 1971, 12, 197.

1973, E, et al, Acta Neurologica Scandinavica,

\section{"Syrup of ipecacuanha"}

SIR,-Your leading article on childhood poisoning (29 November, p 483) deserves wide support for its recommendation that doctors, nurses, and ambulancemen should keep syrup of ipecacuanha at hand. It stores well and works well, but care must be taken to obtain the correct syrup. The American original is ipecac syrup USP. This can readily be made to order, but some pharmacists are not familiar with it. To ask for an unfamiliar preparation is to risk being supplied with ipecacuanha liquid extract $B P$, $15 \mathrm{ml}$ of which would be a massive overdose. Deaths have been caused by this mistake. ${ }^{\text {: }}$ I have heard of a recent occasion when the liquid extract was supplied but was not administered.

The British syrup carries the cumbersome title "ipecacuanha emetic draught, paediatric $B P C$." This is clearly unsuitable for everyday verbal use and a simple name should be made official.

Victoria Hospital

M S FRASER

Kirkcaldy, Fife

1 Smith, R P, and Smith, D M, New England 2 Bates, T, and Grunwaldt, E, American fournal of
${ }_{*}^{*}$ Dr Fraser sent a copy of this letter to the secretary of the BPC Revision Committee, whose reply is printed below.-ED, BMF.

SIR,-Ipecac syrup USP, on which the original work was based, is not readily available in this country, but ipecacuanha emetic draught, paediatric $B P C$ is almost identical and is easily prepared from readily available ingredients using the formula given in the $B P C$. At the time when this preparation was introduced the Codex Revision Committee was aware that misunderstandings had arisen over the meaning of "ipecacuanha syrup" and considered that the use of the term "syrup" for such medicines should be discouraged, as medicated syrups are commonly syrupy stock solutions of drugs for use in extemporaneous preparations. It decided that the title should provide an exact description of the product, the use of which would avoid any possibility of misinterpretation.

At the time of compilation of the $B P C$ 1973 it was considered that the draught should be given only under medical supervision and not included in first-aid kits, and a statement to that effect appears on p 662 . The addition of the preparation to first-aid kits would reinforce the need for a precise title to appear on every bottle so as to avoid any misunderstanding as to the purpose of the contents. However, I agree that there are many occasions when a short title would be useful and I shall see that this is considered when the monograph is revised.

G R BRowN Codex Revision Committee

Pharmaceutical Society of Great Britain London WCl

\section{Streptococcus mutans and dental caries}

SIR,-I read your leading article "Immunisation against dental caries" (22 November, $p$ 424) with personal interest, but I would like to take you up on one small point.

In mentioning the fact that evidence is accumulating that Streptococcus mutans may be associated with dental caries in man you give three references. You then go on to say, "Such a role had been suggested originally in 1924." It was my late father who first described this organism in 1924 when working in the laboratories of $\mathrm{Sir}$ Almroth Wright at St Mary's Hospital with a grant from the Medical Research Council. ${ }^{1} \mathrm{He}$ was attempting to determine the role played by Bacillus acidophilus (one of the lactobacilli thought to be implicated) in the aetiology of dental caries. It was found that $B$ acidophilus could be isolated only from teeth in which cavities were already formed or in which the foci of caries were shallow and contamination from the surface could not be excluded. During the experiments he grew from culture an organism not previously described to which he gave the name Str mutans. This, he found, grew best in a medium with a reaction approximating to that of saliva; it was found in the earliest stages of decay and could be isolated in pure culture from carious dentine which is more or less effectually protected from secondary infection or surface contamination.

Since it is hardly my father's fault that 